\title{
New insight into the mechanisms of ectopic fat deposition improvement after bariatric surgery
}

\author{
Giulia Angelini' ${ }^{1}$, Lidia Castagneto Gissey ${ }^{2}$, Giulia Del Corpo ${ }^{2}$, Carla Giordano ${ }^{3}$, \\ Bruna Cerbelli ${ }^{3}$, Anna Severino ${ }^{1}$, Melania Manco $\mathbb{1}^{4}$, Nicola Basso ${ }^{2}$, \\ Andreas L. Birkenfeld $\mathbb{C}^{5,6,7,8}$, Stefan R. Bornstein ${ }^{5,6,7,8}$, Alfredo Genco ${ }^{2}$, Geltrude Mingrone ${ }^{1,7}$ \\ \& Giovanni Casella ${ }^{2 *}$
}

Non-alcoholic fatty-liver disease (NAFLD) is frequent in obese patients and represents a major risk factor for the development of diabetes and its complications. Bariatric surgery reverses the hepatic features of NAFLD. However, its mechanism of action remains elusive. We performed a comprehensive analysis of the mechanism leading to the improvement of NAFLD and insulin resistance in both obese rodents and humans following sleeve-gastrectomy (SG). SG improved insulin sensitivity and reduced hepatic and monocyte fat accumulation. Importantly, fat accumulation in monocytes was well comparable to that in hepatocytes, suggesting that Plin2 levels in monocytes might be a noninvasive marker for the diagnosis of NAFLD. Both in vitro and in vivo studies demonstrated an effective metabolic regeneration of liver function and insulin sensitivity. Specifically, SG improved NAFLD significantly by enhancing AMP-activated protein kinase (AMPK) phosphorylation and chaperonemediated autophagy (CMA) that translate into the removal of Plin2 coating lipid droplets. This led to an increase in lipolysis and specific amelioration of hepatic insulin resistance. Elucidating the mechanism of impaired liver metabolism in obese subjects will help to design new strategies for the prevention and treatment of NAFLD.

Non-alcoholic fatty liver disease (NAFLD) affects about $25 \%$ of the general population with peaks of $32 \%$ in Middle East and 30\% in South America ${ }^{1}$. A recent study demonstrated a linear relationship between body mass index (BMI) and NAFLD diagnosis, which was about 5- to 9-fold higher in subjects with a BMI between 30 and $32.5 \mathrm{~kg} / \mathrm{m}^{2}$ increasing to ca. 10 - to 14 -fold at BMIs of $37.5-40 \mathrm{~kg} / \mathrm{m}^{2}$ as compared with normal weight subjects ${ }^{2}$. The prevalence of NAFLD in type 2 diabetes (T2D) is estimated to be around $60 \%^{3}$.

Non-alcoholic steatohepatitis (NASH) is a more aggressive form of NAFLD characterized by the presence of inflammatory cell infiltration and hepatocyte ballooning, which may further progress to cirrhosis and/or to hepatocellular carcinoma ${ }^{1}$.

Currently, there are no drugs approved to specifically treat NAFLD or NASH and thus weight loss remains the only approach.

Bariatric surgery (BS) promotes T2D remission both in the short and in the long term ${ }^{4-10}$, as well as remission of both NAFLD and NASH ${ }^{11}$.

The major effect of SG is to reduce energy intake. It is known that a reduced energy intake activates AMP-activated protein kinase (AMPK) in its phosphorylated form that tends to counterbalance a low energy status and restores energy balance through the inhibition of ATP consumption and simultaneous promotion of

${ }^{1}$ Fondazione Policlinico Universitario A. Gemelli IRCCS, Rome, Italy and Università Cattolica del S. Cuore, Rome, Italy. ${ }^{2}$ Department of Surgical Sciences, Sapienza University of Rome, Rome, Italy. ${ }^{3}$ Department of Radiological, Oncological and Pathological Sciences, Sapienza University of Rome, Rome, Italy. ${ }^{4}$ Research Unit for Multi-factorial Diseases, Obesity and Diabetes, Istituti di Ricovero e Cura a Carattere Scientifico, Bambino Gesù Children's Hospital, Rome, Italy. ${ }^{5}$ Department of Medicine III, Universitätsklinikum Carl Gustav Carus an der Technischen Universität Dresden, Dresden, Germany. ${ }^{6}$ Paul Langerhans Institute Dresden of the Helmholtz Center Munich at University Hospital and Faculty of Medicine, TU Dresden, Dresden, Germany. ${ }^{7}$ Diabetes and Nutritional Sciences, King's College London, London, United Kingdom. ${ }^{8}$ Deutsches Zentrum für Diabetesforschung, DZD e.V., Neuherberg, Germany.

*email: giovanni.casella@uniroma1.it 
ATP synthesis ${ }^{12,13}$. Ezquerro et al. ${ }^{14}$ found that in rats under a high-fat diet sleeve gastrectomy improved NAFLD by reducing hepatosteatosis and circulating transaminase levels through the downregulation of lipogenesis and the increase of both autophagy and mitochondrial $\beta$-oxidation.

NAFLD is characterized by the hepatocyte accumulation of neutral lipids forming lipid droplets (LDs), known as hepatic steatosis, surrounded by proteins of which Perilipin 2 (Plin2) is a major component ${ }^{15}$. Plin 2 deletion or suppression of Plin2 expression protect from NAFLD development in genetic models of obesity or in rodents fed a high-fat diet (HFD) $)^{16,17}$.

Chaperone-mediated autophagy (CMA) promotes Plin2 catabolism and, therefore, contributes to LD degradation by facilitating lipolysis ${ }^{18}$. The heat shock cognate protein of $70 \mathrm{kDa}(\mathrm{Hsc} 70)$ recognizes the pentapeptide motif of Plin $2^{19}$ and transports this protein to the lysosome where Plin2 binds to the CMA receptor, LAMP2A (lysosome-associated membrane protein 2A), and is translocated into the lysosomal lumen where is degraded ${ }^{20}$. An important step that allows the binding of Plin2 to LAMP2A is its previous phosphorylation that is dependent on AMPK.

We hypothesize that the energy imbalance generated by SG upregulates liver AMPK and promotes Plin2 degradation leading to the improvement of NAFLD/NASH.

Therefore, we explored these mechanisms performing SG in diet-induced obesity, NAFLD in rats and in subjects with NAFLD.

We observed that ectopic fat deposition in rodents with diet-induced obesity (DIO) included not only hepatic steatosis but also lipid deposition in circulating monocytes in concert with increased Plin 2 expression. We found that SG increased AMPK Thr172 phosphorylation, LAMP2A expression and reduced Plin2 expression in both hepatocytes and monocytes. SG reduced the number of lipid droplets and increased the glycogen storage both in hepatocytes and monocytes; Akt Ser473, Glycogen Synthase Kinase (GSK3 $\alpha \beta)$ Ser21/9 and Forkhead Box O1 (FoxO1) Thr24 phosphorylation were all increased. Plin2 overexpression in primary cultures of hepatocytes and monocytes reduced Akt phosphorylation and lead to insulin resistance.

Together, these data suggest that liver fat accumulation and hepatic insulin resistance share a common pathway coordinated by Plin2.

\section{Results}

Animal study. SG reduces hepatic fat accumulation in diet-induced obesity and significantly reduced Plin2 protein expression in both liver and monocytes. Body weight was significantly lower in the SG than in the sham group ( $534.10 \pm 25.71$ vs. $336.40 \pm 10.31 \mathrm{~g}, \mathrm{P}=0.0003)$. Compared with sham operation, $\mathrm{SG}$ rats drastically reduced fat accumulation in both liver and monocytes of DIO rats (Fig. 1, Panels A-D).

SG significantly reduced Plin2 levels in both liver and monocytes of DIO rats (Liver: $1.70 \pm 0.50$ vs. $0.53 \pm 0.10$, $\mathrm{P}=0.008$; Monocytes: $1.34 \pm 0.50$ vs. $0.24 \pm 0.05, \mathrm{P}=0.01$ ) (Fig. 1, Panels E,F). The Bland-Altman plot in Fig. 1 , Panel G, showed a good agreement between the measures of Plin2 in the liver and in monocytes. AMPK Thr172 phosphorylation and LAMP2A levels were higher in both liver (AMPK Thr172: $0.42 \pm 0.0 .07$ vs. 1.55 \pm 0.17 , $\mathrm{P}=0.0001$; LAMP2A: $0.82 \pm 0.19$ vs. $2.15 \pm 0.47, \mathrm{P}=0.02$ ) (Fig. 1 Panel $\mathrm{H}, \mathrm{I}$ ) and monocytes (AMPK Thr172: $0.54 \pm 0.01$ vs. $1.59 \pm 0.19, \mathrm{P}=0.0001$; LAMP2A: $0.85 \pm 0.16$ vs. $2.60 \pm 0.30, \mathrm{P}=0.0003)$ after $\mathrm{SG}$ as compared with sham operation (Fig. 1 Panel J,K).

SG increased insulin sensitivity influencing Akt, GSK3 $\alpha \beta$ and FoxO1 phosphorylation. The time courses of blood glucose and plasma insulin after the OGTT are shown in Fig. 2, Panels A,B, respectively. Both blood glucose $(73342.50 \pm 8671.01$ vs. $46627.50 \pm 4487.65 \mathrm{mg} / \mathrm{dl} x \mathrm{~min}, \mathrm{P}=0.007)$ and plasma insulin AUCs $(611.31 \pm 186.74$ vs. $371.64 \pm 73.31 \mathrm{ng} / \mathrm{ml} x \mathrm{~min}, \mathrm{P}=0.017$ ) were almost halved in the $\mathrm{SG}$ group as compared with the sham group. Furthermore, we found that Akt Ser473, GSK3 $\alpha \beta$ Ser21/9 and FoxO1 Thr24 phosphorylation were significantly higher in both liver (Akt Ser473: $0.20 \pm 0.04$ vs. $0.51 \pm 0.12, P=0.02$; GSK3 $\alpha$ Ser21: $0.53 \pm 0.12$ vs. $1.31 \pm 0.24$, $\mathrm{P}=0.006$; GSK3 $\beta$ Ser9: $0.19 \pm 0.05$ vs. $0.90 \pm 0.16, \mathrm{P}=0.0003$ : FoxO1 Thr24: $0.56 \pm 0.13$ vs. $1.86 \pm 0.20$, $\mathrm{P}=0.0001$ ) (Fig. 2, Panels C-E) and monocytes (Akt Ser473: $0.17 \pm 0.05$ vs. $0.47 \pm 0.08, \mathrm{P}=0.004$; GSK3 $\alpha$ Ser21: $0.53 \pm 0.12$ vs. $1.31 \pm 0.24, \mathrm{P}=0.006$; GSK33 Ser9: $0.11 \pm 0.03$ vs. $0.40 \pm 0.12, \mathrm{P}=0.03$ : FoxO1 Thr24: $0.23 \pm 0.04$ vs. $0.57 \pm 0.07, \mathrm{P}=0.0006)$ of $\mathrm{DIO}$ rats undergone SG than in sham-operated rats Fig. 2, Panels $\mathrm{F}-\mathrm{H}$ ). Compared with sham operation, SG rats drastically increased glycogen depots in both liver and in monocytes (Fig. 2, Panels I-L).

Human study. The anthropometric characteristics of the obese patients, before and after SG, as well as those of controls are summarized in Table 1 . The weight reduction was ca. $32 \%$ but the weight of the patients remained significantly higher than that of controls. Sleeve gastrectomy improved significantly the lipid profile and reduced significantly transaminases.

Plin2 protein expression in monocytes is significantly reduced after sleeve gastrectomy and strongly correlates with the NAS score. Compared with controls, subjects before SG show high fat accumulation (Fig. 3, Panels A-D). Plin2 protein expression of both liver and monocytes significantly and positively correlated with the NAS score (Liver: $\mathrm{R}=0.52, \mathrm{P}=0.0025$; Monocytes: $\mathrm{R}=0.58, \mathrm{P}=0.00099$ ). Moreover, the expression of Plin2 in monocytes strongly and positively correlated with Plin 2 expression in the liver (Before $S G$ : $R=0.70, P<0.0001$; Controls: $\mathrm{R}=0.90, \mathrm{P}=0.0007$ ).

Figure 3, Panel E shows that Plin2 protein expression in the liver was significantly lower in controls than in obese subjects before SG $(0.76 \pm 0.13$ vs. $0.28 \pm 0.15, \mathrm{P}=0.03)$. Moreover, Plin2 protein expression in monocytes decreased from $0.49 \pm 0.16$ vs. $0.21 \pm 0.04, \mathrm{P}=0.01$ ) after $\mathrm{SG}$ (Fig. 3, Panel F).

AMPK Thr172 phosphorylation and LAMP2A expression in the liver was significantly lower in obese subjects than in controls (AMPK Thr172: $0.31 \pm 0.09$ vs. $1.33 \pm 0.32, \mathrm{P}=0.006$; LAMP2A: $2.23 \pm 0.30$ vs. $5.42 \pm 0.70$, 
E

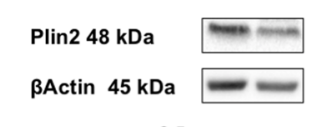

H

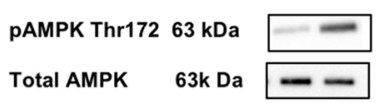

LAMP2A $100 \mathrm{kDa}$

BActin $45 \mathrm{kDa}$

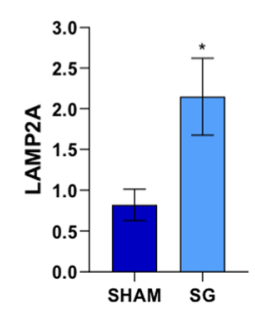

K

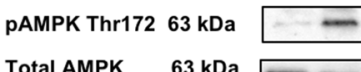

LAMP2A $100 \mathrm{kDa}$

BActin $45 \mathrm{kDa}$

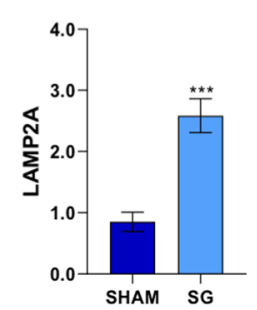

G

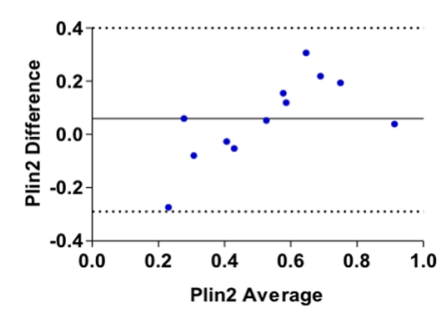

Figure 1. SG reduces hepatic fat accumulation in diet-induced obesity rats and significantly reduced Plin2 protein expression in both liver and monocytes. Panels (A-D): Oil Red O staining of liver biopsies (C,D) and monocytes (E,F) from sham and SG rats; SG drastically reduced hepatic fat accumulation. Panels (E,F): Western blot analysis of Plin2 protein level, in liver biopsies and monocytes of sham and SG rats. SG significantly reduced Plin2 levels in both liver and monocytes of DIO rats (Liver: $1.70 \pm 0.50$ vs. $0.53 \pm 0.10, \mathrm{P}=0.008$; Monocytes: $1.34 \pm 0.50$ vs. $0.24 \pm 0.05, \mathrm{P}=0.01)$. Panel $(\mathrm{G})$ : Bland-Altman plot shows a good agreement between the two measures of Plin2 in the liver and in monocytes. Panels (H-K): SG significantly increased AMPK Thr172 phosphorylation and LAMP2A in both liver (H,I) (AMPK Thr172: 0.42 \pm 0.0 .07 vs. 1.55 \pm 0.17 , $\mathrm{P}=0.0001$; LAMP2A: $0.82 \pm 0.19$ vs. $2.15 \pm 0.47, \mathrm{P}=0.02)$ and monocytes $(\mathrm{J}, \mathrm{K})$ (AMPK Thr $172: 0.54 \pm 0.01$ vs. $1.59 \pm 0.19, \mathrm{P}=0.0001$; LAMP2A: $0.85 \pm 0.16$ vs. $2.60 \pm 0.30, \mathrm{P}=0.0003)$. Data are expressed as mean $\pm \mathrm{SEM}$ $(\mathrm{n}=15$ rat per group).

$\mathrm{P}=0.003$ ) (Fig. 3, Panels G,H). Moreover, as shown in Fig. 3, Panels I,J, AMPK Thr172 phosphorylation and LAMP2A protein expression in monocytes were significantly lower before than after SG (AMPK Thr172: $0.38 \pm 0.04$ vs. $1.28 \pm 0.11, \mathrm{P}=0.0001$; LAMP2A: from $0.37 \pm 0.08$ to $1.00 \pm 0.16, \mathrm{P}=0.003$ ). The Bland-Altman plot in Fig. 3, Panels K,L, shows a good agreement between the measures of Plin2 in the liver and in monocytes of both obese subjects before sleeve gastrectomy and controls. Correlations of different variables with Plin2 are reported in Table 1 of Supplementary Materials.

Akt, GSK $3 \alpha \beta$ and FoxO1 phosphorylation is significantly higher in the liver of controls and increases after sleeve gastrectomy in monocytes. Liver Akt Ser473, GSK3 $\alpha \beta$ Ser21/9, FoxO1 Thr24 phosphorylation were significantly higher in controls than in obese subjects at baseline (Akt Ser473: $0.26 \pm 0.04$ vs $0.96 \pm 0.29, \mathrm{P}=0.01$; GSK3 $\alpha$ Ser21: $1.03 \pm 0.18$ vs. $3.64 \pm 0.97, P=0.02$; GSK3 $\beta$ Ser9: $0.35 \pm 0.13$ vs. $1.98 \pm 0.63, P=0.02$; FoxO1 Thr24: $0.53 \pm 0.06$ vs. $1.49 \pm 017, \mathrm{P}=0.0001$ ) (Fig. 4, Panels A-C). Moreover, Akt Ser473, GSK3 $\alpha \beta$ Ser21/9 and FoxO1 Thr24 phosphorylation in monocytes were increased after SG (Akt Ser473: $0.27 \pm 0.05$ vs. 1.21 \pm 0.29 , $\mathrm{P}=0.005$; GSK3 $\alpha$ Ser21: $0.25 \pm 0.08$ vs. $0.59 \pm 0.20, \mathrm{P}=0.03$; GSK3 $\beta$ Ser9: $0.24 \pm 0.04$ vs. $0.42 \pm 0.07, \mathrm{P}=0.01$; FoxO1 Thr24: $0.29 \pm 0.04$ vs. $0.77 \pm 0.13, \mathrm{P}=0.01$ ) (Fig. 4 , Panels $\mathrm{D}-\mathrm{F}$ ). Compared with controls, subjects before SG show lower glycogen depots in both liver and monocytes (Fig. 4, Panels G-J). 


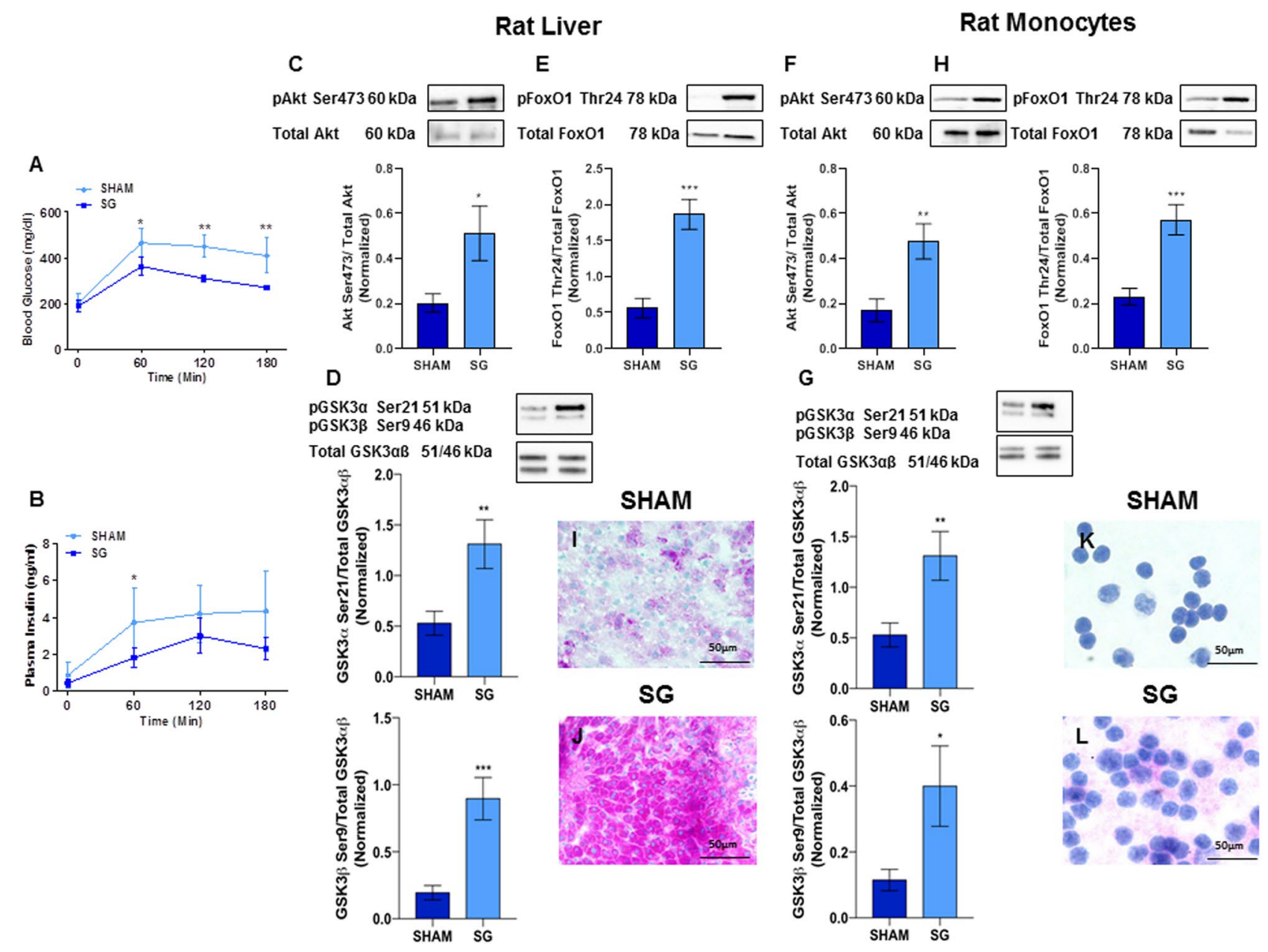

Figure 2. SG significantly improves insulin signaling. Panels (A,B): Time courses of blood glucose (A) and plasma insulin (B) in sham and SG rats. SG significantly reduced both blood glucose and plasma insulin concentrations. $* \mathrm{P}<0.04 ; * * \mathrm{P}=0.004$. Panels $(\mathrm{C}-\mathrm{E})$ : Liver Akt Ser473, GSK3 $\alpha \beta$ Ser21/9 and FoxO1 Thr24 phosphorylation was significantly higher in DIO rats than in SG rats (Akt Ser473: $0.20 \pm 0.04$ vs. $0.51 \pm 0.12$, $\mathrm{P}=0.02$; GSK3 $\alpha$ Ser21: $0.53 \pm 0.12$ vs. $1.31 \pm 0.24, \mathrm{P}=0.006$; GSK3 $\beta$ Ser9: $0.19 \pm 0.05$ vs. $0.90 \pm 0.16$, $\mathrm{P}=0.0003$ : FoxO1 Thr24: $0.56 \pm 0.13$ vs. $1.86 \pm 0.20, \mathrm{P}=0.0001)$. Panels $(\mathrm{F}-\mathrm{H})$ : SG increased monocytes Akt Ser473, GSK3 $\alpha \beta$ Ser21/9 and FoxO1 Thr24 phosphorylation in DIO rats (Akt Ser473: $0.17 \pm 0.05$ vs. $0.47 \pm 0.08, \mathrm{P}=0.004$; GSK3 $\alpha$ Ser2 $1: 0.53 \pm 0.12$ vs. $1.31 \pm 0.24, \mathrm{P}=0.006$; GSK3 $\beta$ Ser9: $0.11 \pm 0.03$ vs. $0.40 \pm 0.12, \mathrm{P}=0.03$ : FoxO1 Thr24: $0.23 \pm 0.04$ vs. $0.57 \pm 0.07, \mathrm{P}=0.0006)$. Panels $(\mathrm{I}-\mathrm{L})$ : Periodic acid-Schiff staining of liver biopsies $(\mathbf{I}, \mathbf{J})$ and monocytes $(\mathbf{K}, \mathbf{L})$ from sham and SG rats; SG markedly increased hepatic glycogen depots. Data are expressed as mean $\pm \operatorname{SEM}(n=15$ rats per group).

In vitro studies. Fat accumulation in primary cultures of hepatocytes and monocytes from healthy controls. To confirm that ectopic fat accumulation occurs also in monocytes and to assess the amount of lipid droplets in primary hepatocytes and monocytes of healthy subjects, cells were exposed to the same concentration of oleic acid for 24 hours. Nile Red was used to stain hepatocytes and monocytes (Fig. 5, Panel A) and flow cytometry was used to quantify the amount of lipid droplet; the Bland-Altman plot showed a good agreement between the amount of lipid droplets in hepatocytes and monocytes (Fig. 5, Panel B).

AKT Ser473 GSK $3 \alpha \beta$ and FoxO1 phosphorylation significantly decrease by overexpressing Plin2 in primary cultures of hepatocytes and monocytes. In order to prove that a high Plin2 expression is responsible for the Akt Ser473, GSK3 $\alpha \beta$ Ser21/9 and FoxO1 Thr24 reduced phosphorylation, we overexpressed Plin2 by transfecting human Plin2 plasmid in primary cultures of hepatocytes and monocytes from controls.

As shown in Fig. 5, Panels C,E,G, Plin2 overexpression significantly reduced Akt Ser473, GSK3 $\alpha \beta$ Ser21/9 and FoxO1 Thr24 phosphorylation in primary cultures of hepatocytes (Akt Ser473: from $1.39 \pm 0.22$ to $0.47 \pm 0.08$, $\mathrm{P}=0.002$; GSK3 $\alpha$ Ser21: from $1.68 \pm 0.29$ to $0.67 \pm 0.16, \mathrm{P}=0.001$; GSK3 $\beta$ Ser9: from $1.29 \pm 0.16$ to $0.61 \pm 0.08$, $\mathrm{P}=0.0001$; FoxO1 Thr24: from $0.61 \pm 0.09$ to $0.33 \pm 0.04, \mathrm{P}=0.001$. Additionally, Plin2 overexpression significantly reduced Akt Ser473, GSK3 $\alpha \beta$ Ser21/9 and FoxO1 Thr24 phosphorylation in monocytes of healthy controls (Akt Ser473: from $1.03 \pm 0.25$ to $0.17 \pm 0.03, \mathrm{P}=0.008$; GSK3 $\alpha$ Ser21: from $1.24 \pm 0.34$ to $0.46 \pm 0.04, \mathrm{P}=0.04$; GSK3 $\beta$ Ser9: from $1.85 \pm 0.28$ to $0.78 \pm 0.01, \mathrm{P}=0.002$; FoxO1 Thr24: from $1.20 \pm 0.36$ to $0.46 \pm 0.11, \mathrm{P}=0.02$ 


\begin{tabular}{|l|l|l|l|l|l|l|}
\hline & $\begin{array}{l}\text { Before } \\
\text { Metabolic } \\
\text { Surgery }\end{array}$ & $\begin{array}{l}\text { P value } \\
\text { Before/After }\end{array}$ & $\begin{array}{l}\text { After Metabolic } \\
\text { Surgery }\end{array}$ & $\begin{array}{l}\text { P value Before } \\
\text { Surgery vs. } \\
\text { Controls }\end{array}$ & Controls & $\begin{array}{l}\text { P value After } \\
\text { Surgery vs. } \\
\text { Controls }\end{array}$ \\
\hline Number of patients & 15 & & 15 & & 8 & \\
\hline Age (years) & $39.7 \pm 2.1$ & NS & $40.67 \pm 2.1$ & NS & $39.9 \pm 2.9$ & NS \\
\hline Weight $(\mathrm{kg})$ & $117.4 \pm 4.8$ & $<0.0001$ & $79.6 \pm 2.1$ & $<0.0001$ & $64.7 \pm 3.2$ & 0.0006 \\
\hline BMI $\left(\mathrm{kg} / \mathrm{m}^{2}\right)$ & $43.0 \pm 0.9$ & $<0.0001$ & $29.2 \pm 0.7$ & $<0.0001$ & $21.5 \pm 0.74$ & $<0.0001$ \\
\hline Plasma Glucose $(\mathrm{mg} / \mathrm{dl})$ & $94.1 \pm 2.3$ & 0.01 & $86.7 \pm 1.4$ & $<0.0001$ & $74.8 \pm 2.1$ & $<0.0001$ \\
\hline Plasma Insulin $(\mathrm{mU} / \mathrm{l})$ & $16.0 \pm 3.7$ & 0.02 & $6.1 \pm 1.3$ & $\mathrm{NS}$ & $8.2 \pm 0.62$ & NS \\
\hline HOMA-IR & $3.6 \pm 0.8$ & 0.02 & $1.3 \pm 0.3$ & 0.02 & $1.5 \pm 0.11$ & NS \\
\hline HDL-cholesterol $(\mathrm{mg} / \mathrm{dl})$ & $40.0 \pm 4.3$ & 0.01 & $56.0 \pm 4.4$ & 0.0008 & $64.6 \pm 4.8$ & NS \\
\hline *LDL-cholesterol $(\mathrm{mg} / \mathrm{dl})$ & $137.3 \pm 8.6$ & 0.0002 & $93.9 \pm 5.7$ & 0.0004 & $95.2 \pm 4.0$ & NS \\
\hline Triglycerides $(\mathrm{mg} / \mathrm{dl})$ & $146.2 \pm 15.6$ & 0.0003 & $72.3 \pm 7.7$ & 0.03 & $105.7 \pm 8.4$ & 0.007 \\
\hline AST $(\mathrm{IU} / \mathrm{l})$ & $57.5 \pm 4.7$ & $<0.0001$ & $29.2 \pm 1.7$ & $<0.0001$ & $18.6 \pm 1.8$ & NS \\
\hline ALT $(\mathrm{IU} / \mathrm{l})$ & $32.3 \pm 5.4$ & 0.02 & $18.5 \pm 1.16$ & 0.02 & $17.7 \pm 0.63$ & NS \\
\hline NAS score & $6.07 \pm 0.63$ & & & & & \\
\hline Excess Weight $(\mathrm{g})$ & $49.57 \pm 3.35$ & $<0.0001$ & $11.76 \pm 1.79$ & & & \\
\hline Excess Weight Loss (\%) & & & $75.63 \pm 3.42$ & & & \\
\hline
\end{tabular}

Table 1. Clinical characteristics of the study population (mean \pm SEM.). ${ }^{*} \mathrm{LDL}$-cholesterol $\left(\mathrm{mg} \mathrm{dL}^{-1}\right)=$ total cholesterol $\left(\mathrm{mg} \mathrm{dL}^{-1}\right)$ - HDL-cholesterol $\left(\mathrm{mg} \mathrm{dL}^{-1}\right)$ - triglycerides $\left(\mathrm{mg} \mathrm{dL}^{-1}\right) / 5$.

(Fig. 5, Panels D,F,H). Supplementary Fig. 1, Panels A,C, shows the efficiency of cell transfection with pmaxGFP vector and Plin2 overexpression by flow cytometry (Supplementary Fig. 1, Panels B,D).

Correlations. We did not find significant correlations between changes in the phosphorylation of protein of interest, namely, AMPK Thr172, LAMP2A, Akt Ser473, GSK3 $\alpha \beta$ Ser21/9 and FoxO1 Thr24 and changes in body weight in both humans and rodents.

Liver insulin sensitivity improvement induced by metformin or pioglitazone reduces Plin2 levels in primary hepatocyte cultures via CMA activation. Human and rat primary hepatocytes were cultured for $24 \mathrm{~h}$ in the presence of high levels of oleic acid, glucose and insulin to induce insulin resistance. Figure 6 shows that both metformin and pioglitazone at pharmacological concentrations decreased Plin2 expression by promoting AMPK phosphorylation and consequent activation of CMA. Indeed, AMPK phosphorylation was increased after pioglitazone or metformin exposure (pioglitazone in human primary hepatocytes: from $0.43 \pm 0.04$ to $1.18 \pm 0.26, \mathrm{P}=0.04$; metformin in human primary hepatocytes: from $0.43 \pm 0.04$ to $0.94 \pm 0.12, \mathrm{P}=0.005$; pioglitazone in rat primary hepatocytes: from $0.81 \pm 0.11$ to $1.87 \pm 0.31, \mathrm{P}=0.03$; metformin in rat primary hepatocytes: from $0.81 \pm 0.11$ to $1.50 \pm 0.14, \mathrm{P}=0.01$ ) (Fig. 6, Panels A,B).

Pioglitazone or metformin increased LAMP2A expression (pioglitazone in human primary hepatocytes: from $2.16 \pm 0.21$ to $3.40 \pm 0.37, \mathrm{P}=0.02$; metformin in human primary hepatocytes: from $2.16 \pm 0.21$ to $3.30 \pm 0.36$, $\mathrm{P}=0.04$; pioglitazone in rat primary hepatocytes: from $2.91 \pm 0.70$ to $4.92 \pm 0.90, \mathrm{P}=0.02$; metformin in rat primary hepatocytes: from $2.91 \pm 0.70$ to $5.62 \pm 1.11, \mathrm{P}=0.04$ ) (Fig. 6, Panels C,D).

In contrast, pioglitazone or metformin significantly decreased Plin2 expression (pioglitazone in human primary hepatocytes: from $0.67 \pm 0.13$ to $0.38 \pm 0.08, \mathrm{P}=0.02$; metformin in human primary hepatocytes: from $0.67 \pm 0.13$ to $0.30 \pm 0.06, \mathrm{P}=0.02$; pioglitazone in rat primary hepatocytes: from $0.65 \pm 0.11$ to $0.41 \pm 0.11$, $\mathrm{P}=0.03$; metformin in rat primary hepatocytes: from $0.65 \pm 0.11$ to $0.39 \pm 0.06, \mathrm{P}=0.02$ ) (Fig. 6 , Panels $\mathrm{E}, \mathrm{F}$ ).

Moreover, Nile Red staining shows a reduction in lipid droplet accumulation after both pioglitazone (Fig. 7, Panel C) and metformin (Fig. 7, Panel D) exposure in human hepatocytes primary cultures.

\section{Discussion}

Non-alcoholic fatty-liver disease (NAFLD) is currently the most frequent liver disorder in the Western world ${ }^{1}$. Diabetes is a major risk factor for the development of NAFLD. Currently, there are no FDA-approved drugs specifically tailored for NAFLD or NASH. Bariatric/metabolic surgery is effective in reversing the hepatic features of NAFLD and NASH, as shown by retrospective or prospective but non randomized studies ${ }^{11}$. However, the mechanism of action of bariatric/metabolic surgery remains elusive.

In the present study, we provide evidences both in rodents and in humans that after SG there is a net increase of phosphorylated AMPK and LAMP2A expression, causing a decrease in Plin2 expression and a reduction in LDs accumulation in both liver and circulating monocytes. In addition, we observed an increased phosphorylation of Akt Ser473, GSK3 $\alpha \beta$ Ser21/9 and FoxO1 Thr24 and an increased glycogen deposition in liver and monocytes of both rodents and humans after SG.

We also report that fat accumulation in monocytes was well comparable to that in hepatocytes, suggesting that Plin2 levels in monocytes might be a non-invasive marker for the diagnosis of NAFLD.

The changes in phosphorylation of proteins of interest were independent of changes in body weight both in humans and in rats suggesting a weight-independent mechanism of action of SG. Most beneficial effects of sleeve gastrectomy are, in fact, achieved in a weight-independent manner as shown by Frühbeck ${ }^{21}$, although 


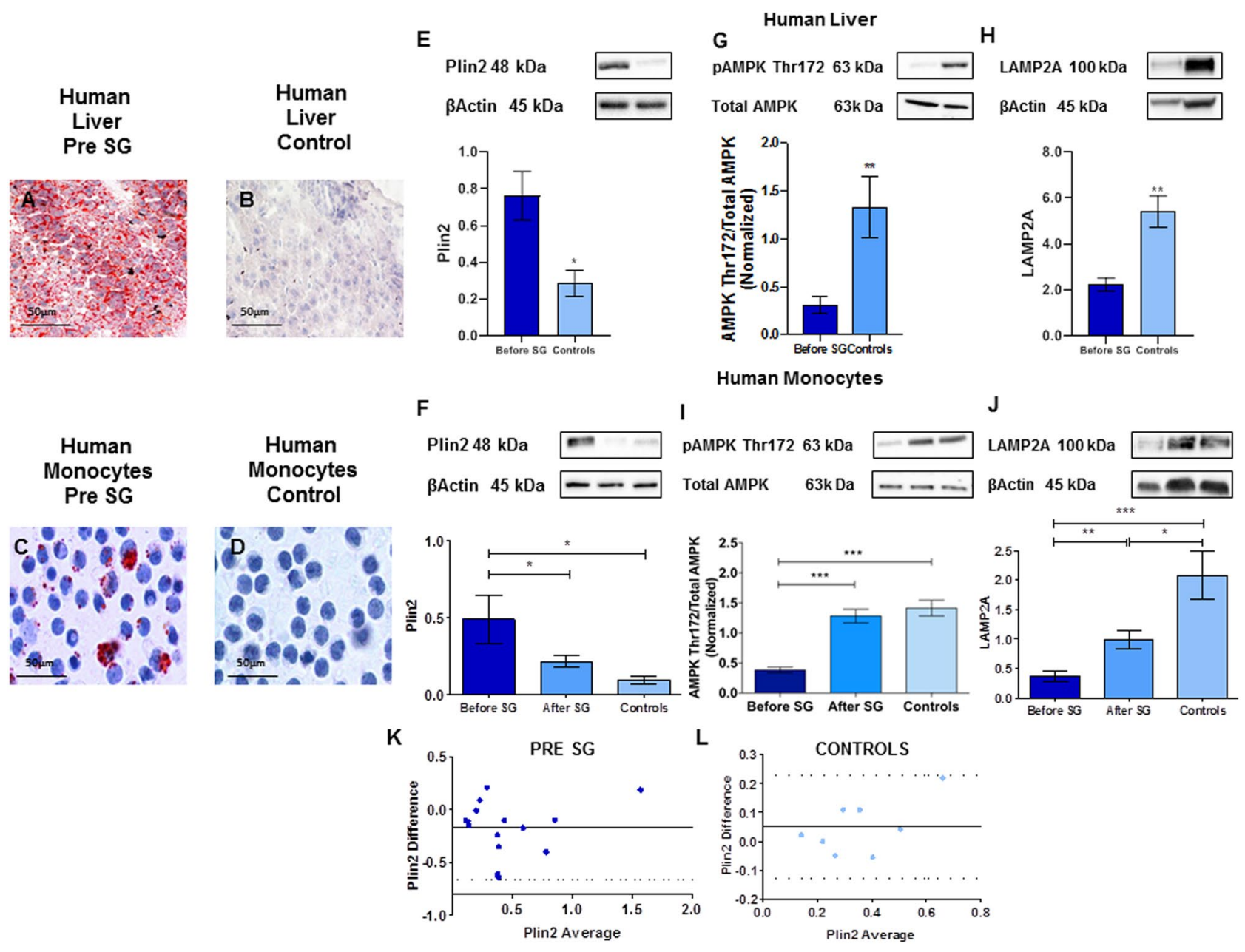

Figure 3. Plin2 protein expression in monocytes was significantly reduced after sleeve gastrectomy. Panels (A-D): Oil Red O staining of liver biopsies $(\mathbf{A}, \mathbf{B})$ and monocytes $(\mathbf{C}, \mathbf{D})$ of obese subjects with NAFLD before sleeve gastrectomy and controls. Fat accumulation was much higher in obese subjects than in controls. Panel (E): Plin2 protein expression in the liver was significantly lower in controls than in obese subjects with NAFLD $(0.76 \pm 0.13$ vs. $0.28 \pm 0.15, \mathrm{P}=0.03)$. Panel $(\mathrm{F})$ : Plin2 protein expression in monocytes decreased from $0.49 \pm 0.16$ vs. $0.21 \pm 0.04, \mathrm{P}=0.01$ after sleeve gastrectomy. Panels $(\mathrm{G}, \mathrm{H})$ : AMPK Thr172 phosphorylation and LAMP2A expression in the liver was significantly lower in obese subjects with NAFLD than in controls (AMPK Thr172: $0.31 \pm 0.09$ vs. $1.33 \pm 0.32, \mathrm{P}=0.006$; LAMP2A: $2.23 \pm 0.30$ vs. $5.42 \pm 0.70, \mathrm{P}=0.003)$. Panels $(\mathrm{I}, \mathrm{J})$ : AMPK Thr172 phosphorylation and LAMP2A protein expression in monocytes were significantly lower before than after SG (AMPK Thr172: $0.38 \pm 0.04$ vs. $1.28 \pm 0.11, \mathrm{P}=0.0001$; LAMP2A: from $0.37 \pm 0.08$ to $1.00 \pm 0.16$, $\mathrm{P}=0.003)$. Panels $(\mathrm{K}, \mathrm{L})$ : Bland-Altman plots show a good agreement between the measures of Plin 2 in liver and in monocytes of obese subjects with NAFLD and controls. Data are expressed as mean \pm SEM ( $n=15$ obese subjects with NAFLD before and after SG and $\mathrm{n}=8$ controls).

the mechanism of action is not completely elucidated. One of the pathways affected by caloric restriction is the AMPK one. AMPK controls glucose disposal by inhibiting liver gluconeogenesis and increasing muscle glucose uptake $^{22-25}$. Targeting hepatic AMPK with an AMPK-specific small-molecule activator reduces liver steatosis and improves glucose disposal in obese mice ${ }^{26,27}$. Recently, Garcia et al. ${ }^{28}$ showed that liver-specific AMPK activation in DIO mice protects against liver steatosis.

Our data show a net increase of AMPK phosphorylation after SG in both hepatocytes and monocytes either in rodents or in humans. AMPK phosphorylation is a crucial step for the binding of Plin2 to LAMP2A that leads to an increased Plin 2 catabolism and lipid droplets exposure to lypolysis ${ }^{19}$. Indeed, after SG we found a significant reduction of Plin2 expression and lipid droplets accumulation.

Bariatric/metabolic surgery procedures that reduce caloric intake, including SG, have shown high rates of remission of type 2 diabetes mostly due to the improvement of insulin sensitivity ${ }^{7}$. In fact, here we report a significant increase of Akt Ser473, GSK3 $\alpha \beta$ Ser21/9 and FoxO1 Thr24 phosphorylation in both liver and monocytes, which points towards an improved hepatic insulin sensitivity with reduced gluconeogenesis and increased glycogen synthesis. Indeed, the inhibition of GSK $\alpha \beta$ activity through its phosphorylation leads to increased glycogen synthesis, while the inhibition of FoxO1 activity, again through its phosphorylation, reduces gluconeogenesis ${ }^{29}$. 


\section{Human Liver}

A

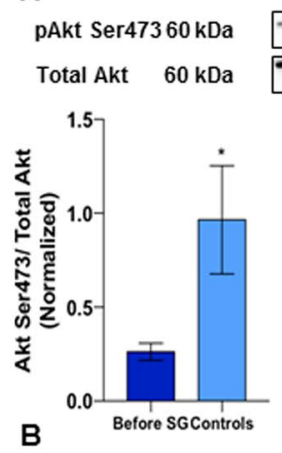

pGSK3a Ser2151 kDa pGSK3 3 Ser9 $46 \mathrm{kDa}$

Total GSK3aß $51 / 46 \mathrm{kDa}$
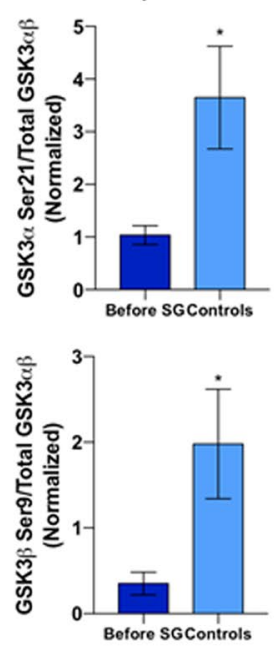

C
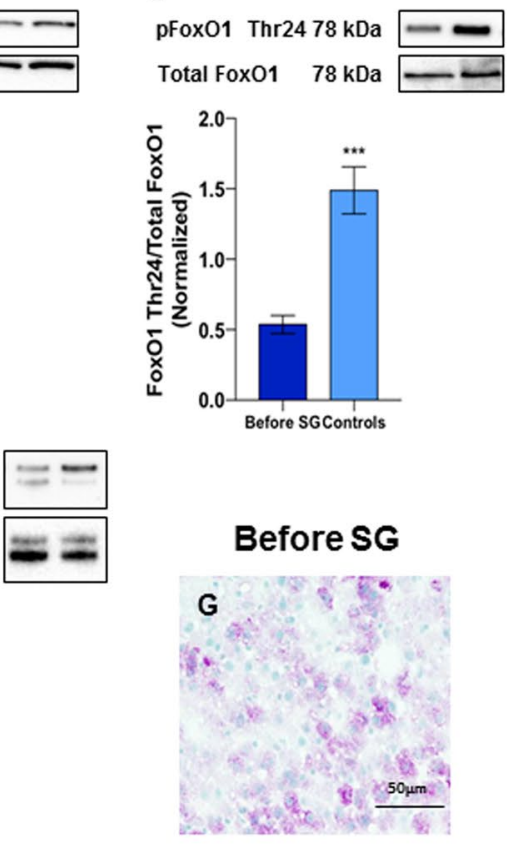

Control

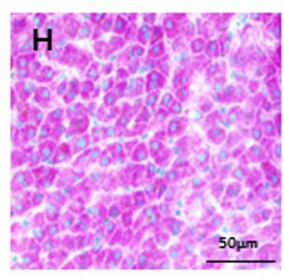

Human Monocytes

D

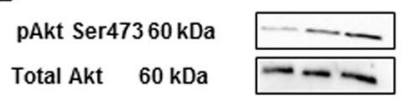

$F$

pFox01 Thr24 78 kDa

Total FoxO1 $78 \mathrm{kDa}$

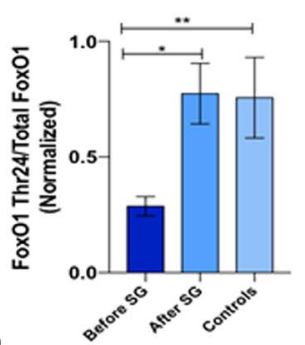

E
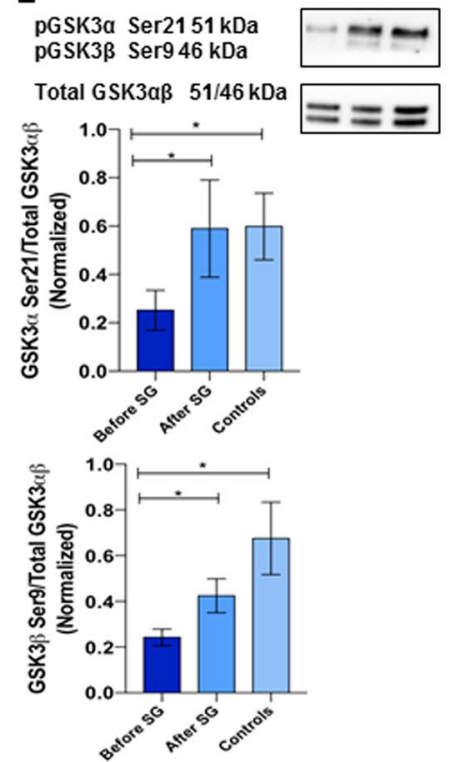

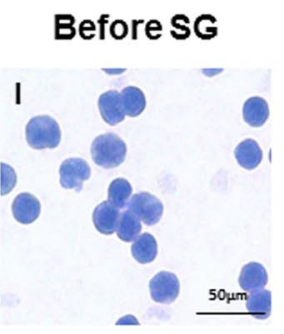

Control

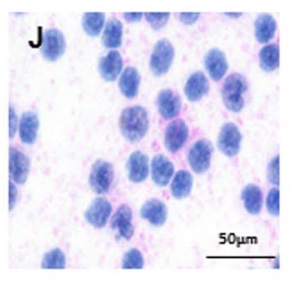

Figure 4. SG significantly affects insulin signaling. Panels (A-C): Liver Akt Ser473, GSK3 $\alpha \beta$ Ser21/9, FoxO1 Thr24 phosphorylation was significantly higher in controls than in obese subjects with NAFLD (Akt Ser473: $0.26 \pm 0.04$ vs $0.96 \pm 0.29, \mathrm{P}=0.01$; GSK $3 \alpha$ Ser21: $1.03 \pm 0.18$ vs. $3.64 \pm 0.97, \mathrm{P}=0.02$; GSK $3 \beta$ Ser9: $0.35 \pm 0.13$ vs. $1.98 \pm 0.63, \mathrm{P}=0.02$; FoxO1 Thr24: $0.53 \pm 0.06$ vs. $1.49 \pm 017, \mathrm{P}=0.001)$ ). Panels (D-F): Akt Ser473, GSK3 $\alpha \beta$ Ser21/9 and FoxO1 Thr24 phosphorylation in monocytes is increased after SG (Akt Ser473: 0.27 \pm 0.05 vs. $1.21 \pm 0.29, \mathrm{P}=0.005$; GSK $3 \alpha$ Ser21: $0.25 \pm 0.08$ vs. $0.59 \pm 0.20, \mathrm{P}=0.03$; GSK3 $\beta$ Ser9: $0.24 \pm 0.04$ vs. $0.42 \pm 0.07, \mathrm{P}=0.01$; FoxO1 Thr24: $0.29 \pm 0.04$ vs. $0.77 \pm 0.13, \mathrm{P}=0.001)$. Panels $(\mathrm{G}-\mathrm{J})$ : Periodic acid-Schiff staining of liver biopsies $(\mathbf{G}, \mathbf{H})$ and monocytes $(\mathbf{I}, \mathbf{J})$ of obese subjects with NAFLD before sleeve gastrectomy and controls. Glycogen stores were much higher in controls than in obese subjects. Data are expressed as mean \pm SEM ( $n=15$ obese subjects with NAFLD before and after SG and $n=8$ controls).

These results are supported by in vitro data showing that overexpression of Plin2, using a human-Plin2 plasmid in primary hepatocyte and monocyte cultures, significantly affects insulin signaling decreasing Akt Ser473, GSK3 $\alpha \beta$ Ser21/9 and FoxO1 Thr24 phosphorylation.

Several studies have shown that AMPK is activated by two major antidiabetic drugs, metformin and pioglitazone $^{19,30}$. We report that treatment of primary hepatocytes with pioglitazone or metformin reduces Plin 2 expression, LDs accumulation and improves insulin signaling.

When insulin binds rodent or human hepatocytes it is internalized and preferentially associated with lysosomal structures ${ }^{20,31}$. The upregulation of LAMP2A we found might, thus, reflect the improvement of hepatic insulin resistance after sleeve gastrectomy that was shown by the significant reduction of HOMA-IR values.

The lack of post-surgery liver biopsies and the lack of a pair-fed group of rats represent two limitations of our study, although our results in humans are supported by the similar findings associated with SG in rodents. We showed, in fact, a high correlation between LDs and Plin 2 content in the liver and in monocytes that were available also after SG. Furthermore, the surrogate marker HOMA-IR, that has been shown to be highly correlated with hepatic insulin resistance as assessed by the gold standard euglycemic hyperinsulinemic clamp ${ }^{32}$, was used to assess hepatic insulin resistance.

In conclusion, we propose that excessive caloric intake, which is associated with NAFLD and obesity, reduces AMPK activation, causing a decrease of Plin2 catabolism mediated by chaperone-mediated autophagy that leads to an increase of ectopic fat deposition and simultaneously to hepatic insulin resistance. After SG, the 
A

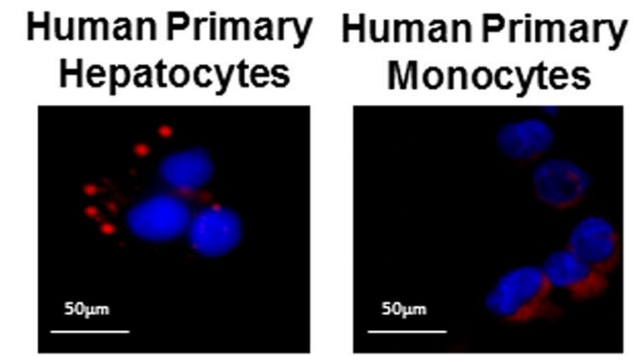

Human Primary Hepatocytes

C pAkt Ser47360 kDa Total Akt $60 \mathrm{kDa}$

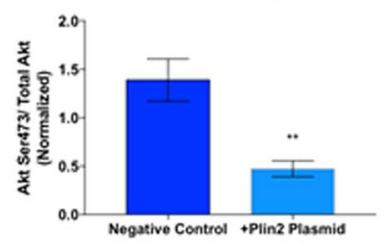

E

pGSK3a Ser2151 kDa pGSK3 $\beta$ Ser9 46 kDa

Total GSK3aß $51 / 46 \mathrm{kDa}$
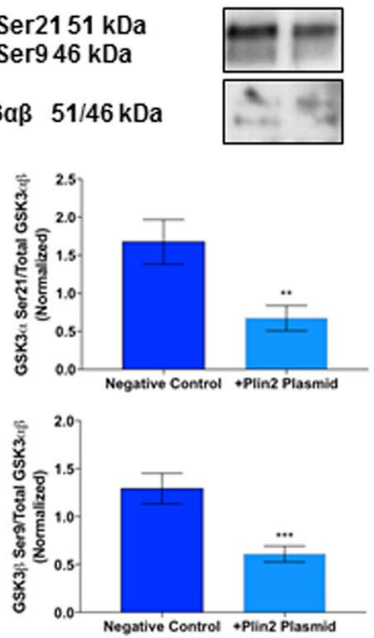

G
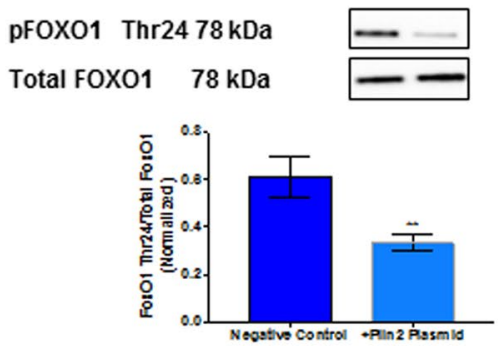

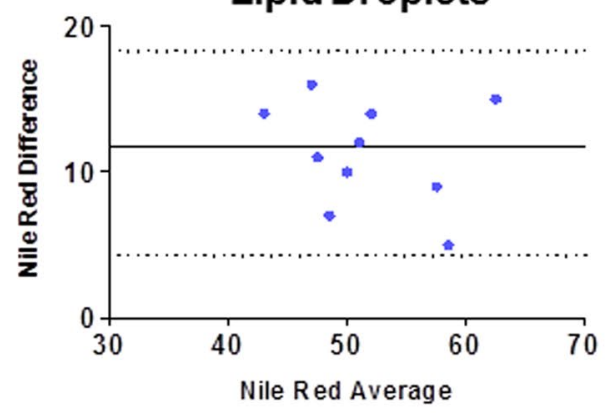

\section{Human Primary Monocytes}

D
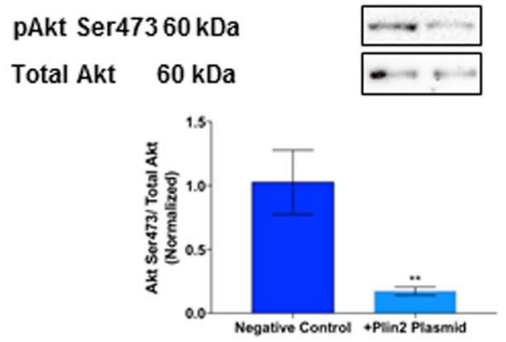

$\mathbf{F}$
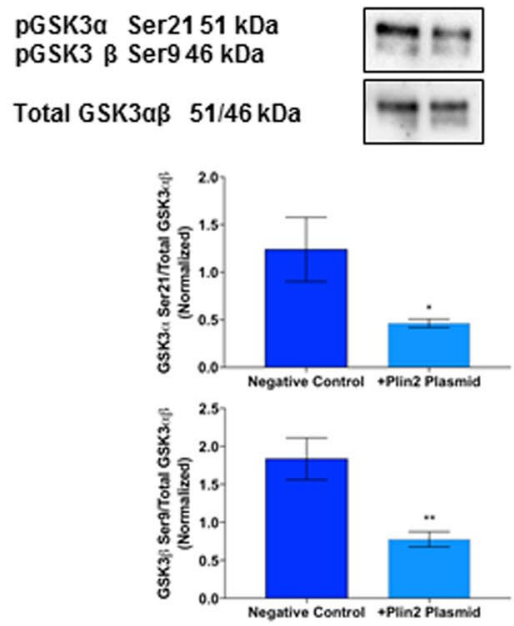

H

pFOXO1 Thr24 $78 \mathrm{kDa}$
Total FOX01 $78 \mathrm{kDa}$
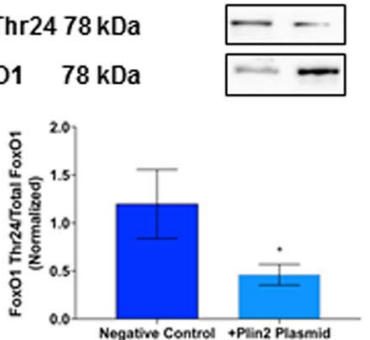

Figure 5. Overexpression of Plin2 decreases AKT Ser473, GSK3 $\alpha \beta$ Ser21/9 and FoxO1 Thr24 phosphorylation. Panel (A): Nile Red staining of liver biopsies and monocytes. Nile Red staining shows that ectopic fat accumulation occurs not only in hepatocytes but also in monocytes. Panel (B): Bland-Altman plot shows a good agreement between the measures of lipid droplets accumulation in hepatocytes and monocytes from healthy controls stimulated with oleic acid. Panels (C,D): Plin2 overexpression significantly reduced Akt Ser473 phosphorylation in hepatocytes $(\mathbf{C})$, from $1.39 \pm 0.22$ to $0.47 \pm 0.08, \mathrm{P}=0.002$ and $(\mathrm{D})$ in monocytes, from $1.03 \pm 0.25$ to $0.17 \pm 0.03, P=0.008$. Panels (E,F): Plin 2 overexpression significantly reduced GSK $3 \alpha$ Ser 21 phosphorylation in hepatocytes $(\mathbf{E})$ from GSK3 $\alpha$ Ser21: from $1.68 \pm 0.29$ to $0.67 \pm 0.16, P=0.001$; GSK3 $\beta$ Ser9: from $1.29 \pm 0.16$ to $0.61 \pm 0.08, \mathrm{P}=0.0001$ and in monocytes $(\mathbf{F}), \mathrm{GSK} 3 \alpha$ Ser21: from $1.24 \pm 0.34$ to $0.46 \pm 0.04, \mathrm{P}=0.04$; GSK3 3 Ser9: from $1.85 \pm 0.28$ to $0.78 \pm 0.01, \mathrm{P}=0.002$. Panels $(\mathrm{G}, \mathrm{H})$ : Plin2 overexpression significantly reduced FoxO1 Thr24 in hepatocytes $(\mathbf{G})$ from 0 FoxO1 Thr24: from $0.61 \pm 0.09$ to $0.33 \pm 0.04$, $\mathrm{P}=0.001$ and in monocytes $(\mathbf{H})$ from FoxO1 Thr24: from $1.20 \pm 0.36$ to $0.46 \pm 0.11, \mathrm{P}=0.02$. Data are expressed as mean \pm SEM ( $\mathrm{n}=5$ controls). 


\section{Human Primary Hepatocytes}
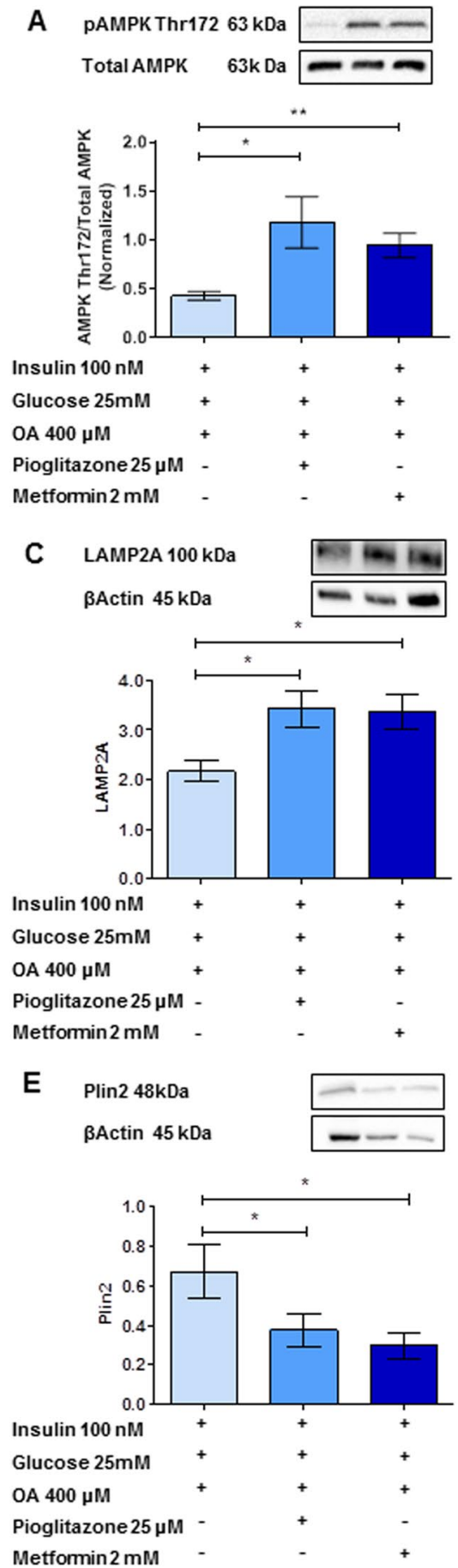

\section{Rat Primary Hepatocytes}
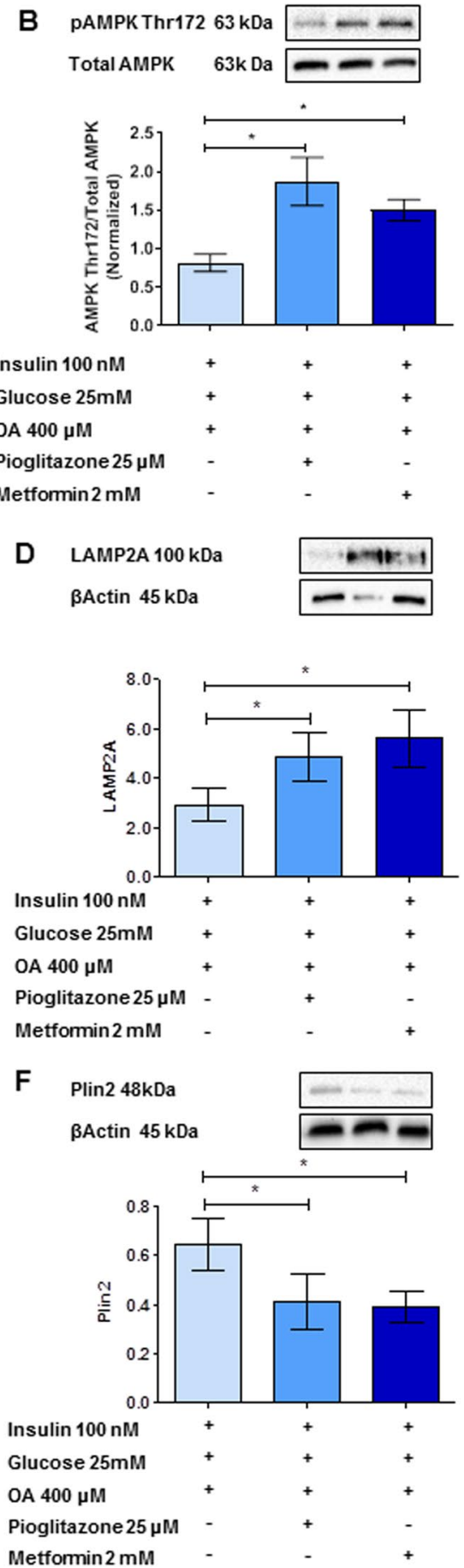

Figure 6. Pioglitazone or metformin reduced Plin2 levels in primary hepatocyte cultures via CMA activation. Panels (A,B): Pioglitazone and metformin at pharmacological concentrations significantly increased AMPK Thr172 phosphorylation, (pioglitazone in human primary hepatocytes: from $0.43 \pm 0.04$ to $1.18 \pm 0.26$, $\mathrm{P}=0.04$; metformin in human primary hepatocytes: from $0.43 \pm 0.04$ to $0.94 \pm 0.12, \mathrm{P}=0.005$; pioglitazone in rat primary hepatocytes: from $0.81 \pm 0.11$ to $1.87 \pm 0.31, \mathrm{P}=0.03$; metformin in rat primary hepatocytes: from $0.81 \pm 0.11$ to $1.50 \pm 0.14, \mathrm{P}=0.01$ ). Panels $(\mathrm{C}, \mathrm{D})$ : Pioglitazone and metformin at pharmacological concentrations significantly increased LAMP2A protein expression (pioglitazone in human primary hepatocytes: from $2.16 \pm 0.21$ to $3.40 \pm 0.37, \mathrm{P}=0.02$; metformin in human primary hepatocytes: from $2.16 \pm 0.21$ to $3.30 \pm 0.36, \mathrm{P}=0.04$; pioglitazone in rat primary hepatocytes: from $2.91 \pm 0.70$ to $4.92 \pm 0.90$, $\mathrm{P}=0.02$; metformin in rat primary hepatocytes: from $2.91 \pm 0.70$ to $5.62 \pm 1.11, \mathrm{P}=0.04)$. Panels $(\mathrm{E}, \mathrm{F})$ :

Pioglitazone and metformin at pharmacological concentrations significantly decreased Plin2 protein expression 
(pioglitazone in human primary hepatocytes: from $0.67 \pm 0.13$ to $0.38 \pm 0.08, \mathrm{P}=0.02$; metformin in human primary hepatocytes: from $0.67 \pm 0.13$ to $0.30 \pm 0.06, \mathrm{P}=0.02$; pioglitazone in rat primary hepatocytes: from $0.65 \pm 0.11$ to $0.41 \pm 0.11, \mathrm{P}=0.03$; metformin in rat primary hepatocytes: from $0.65 \pm 0.11$ to $0.39 \pm 0.06$, $\mathrm{P}=0.02)$. Data are expressed as mean $\pm \operatorname{SEM}(\mathrm{n}=5$ controls $)$.

energy imbalance due to reduced caloric intake causes an increase in AMPK phosphorylation, a crucial step in Plin2-LAMP2A binding, leading to an enhanced Plin2 autophagy that exposes LD triglycerides to intracellular lipases. In addition, AMPK increased phosphorylation improves insulin signaling and promotes glycogen synthesis (Fig. 8).

\begin{abstract}
Methods
Animal model. Thirty Wistar rats of both sexes aged 10 weeks were housed individually in a controlled room at $22^{\circ} \mathrm{C}$ with a 12 -h day/night cycle (lights on $0700-1900 \mathrm{~h}$ ). The animals received a purified tripalmitin-based High fat liquid diet (HFD) ad libitum (Rieper AG, Bolzano, Italy) supplying $71 \%$ of energy from saturated fat, although corn oil (1.9/100 g diet) was present in order to prevent essential fatty acid deficiency, $20 \%$ from carbohydrate comprising cornstarch and sucrose (2:1 weight for weight) and $10 \%$ from protein. The HFD was continued for 10 weeks before and 15 weeks after the operation. The animals randomly underwent SG or sham operation. Survival rates were $90 \%$ after sham operation, and $75 \%$ after SG. All experimental procedures were approved by the Catholic University of Rome Institutional Animal Care Committee and all methods were performed in accordance with the relevant guidelines and regulations ${ }^{33}$.
\end{abstract}

Interventions. The rats were anesthetized using ketamine $(75 \mathrm{mg} / \mathrm{kg}$ intramuscularly) and xylazine $(10 \mathrm{mg} /$ $\mathrm{kg}$ intramuscularly). Ten milliliters of sterile $0.9 \% \mathrm{NaCl}$ were administered subcutaneously before surgery. Access to the peritoneal cavity was obtained by $3-\mathrm{cm}$ laparotomy ${ }^{33}$.

Vertical sleeve gastrectomy. A laparotomy incision was made in the abdominal wall and the stomach was isolated outside the abdominal cavity and placed on saline-moistened gauze pads. The gastric to spleen connections were released along the greater curvature. The lateral $80 \%$ of the stomach was excised leaving a tubular gastric remnant in continuity with the esophagus and with the pylorus and duodenum. After the operation, the abdominal wall was closed in layers ${ }^{33}$.

In sham-operated rats, a midline laparotomy was performed, and the stomach was exposed and gently manipulated. The abdominal cavity was kept open for the same amount of time required to perform the other operations. A 1-cm gastrotomy was performed and then closed as in the SG group.

Postoperative care. At the end of the surgical procedures, all rats received sterile $0.9 \% \mathrm{NaCl} 10 \mathrm{~mL}$ i.p. and $10 \mathrm{~mL}$ s.c. to maintain hydration during healing. The animals received ketoprofen $5 \mathrm{mg} / \mathrm{kg}$ as an analgesic. They were placed on a heated mat until they recovered and then were returned to their home cages. The rats were allowed to drink purified water for $12 \mathrm{~h}$ after surgery, and a liquid diet containing $5 \%$ glucose and $0.2 \% \mathrm{KCl}$ was provided for the next $48 \mathrm{~h}$. Thereafter, they received the HFD until 15 weeks after surgery ${ }^{34}$.

Oral glucose tolerance test. The oral glucose tolerance test (OGTT) was performed at kill. Animals were fasted overnight and then received a $50 \% \mathrm{D}$-glucose solution $(1 \mathrm{~g} / \mathrm{kg}$ body weight) by oral gavage. Blood was collected from the tail vein for measurement of glucose and insulin concentrations at $0,15,30,60,90,120$ and $180 \mathrm{~min}$ at the end of the study. After centrifugation, plasma was divided into appropriate subsamples and stored at $-20^{\circ} \mathrm{C}$ until analyses.

Analytical methods. Blood glucose levels were analyzed by glucometer (Accu-Chek, Roche Diagnostics Division, Grenzacherstrasse, Switzerland). Serum insulin was measured by a rat insulin ultrasensitive ELISA (Biovendor $\mathrm{GmbH}$, Kassel, Germany), with a sensitivity of $0.025 \mathrm{ng} / \mathrm{ml}$ and an intra- and inter-assay precision of $10 \%$.

Hepatocytes isolation. Hepatocytes were isolated by collagenase perfusion ${ }^{35}$ of the liver. Cells were cultured for 24 hours in DMEM with 10\% FBS.

\title{
Human Studies
}

Study population. We enrolled 15 obese subjects with NAFLD of both sexes (age 39.7 \pm 2.1 ) before and one year after laparoscopic SG. Eight subjects who underwent laparoscopic elective cholecystectomy, but otherwise in healthy conditions, served as controls. Venous blood samples were collected, after 12 hours fasting, at the time of the patients' enrolment and one year after SG.

Comprehensive history was obtained from all subjects. Physical exam, including height, weight and anthropometric measurements were conducted. Body mass index (BMI) was calculated by dividing the weight (kg) by height $(\mathrm{cm})$. A detailed medication list was obtained. All participants underwent biochemical tests: ALT, AST, fasting glucose, insulin, triglycerides, HDL and LDL-cholesterol.

Exclusion criteria were ${ }^{1}$ : regular and/or excessive alcohol use $(>20 \mathrm{~g}$ of alcohol per day for women and $>30 \mathrm{~g}$ of alcohol per day for men $)^{2}$; clinically evidence of secondary NALFD due to iatrogenic gastrointestinal disorder or immune deficiency (HIV) infection ${ }^{3}$; clinically evidence of non-NAFLD liver disease including hepatitis B or $\mathrm{C}$, hemochromatosis ${ }^{4}$, Wilson disease ${ }^{5}$, glycogen storage disease ${ }^{6}$, alpha-1antitrypsin deficiency ${ }^{7}$, autoimmune 


\section{Untreated Human primary hepatocytes}
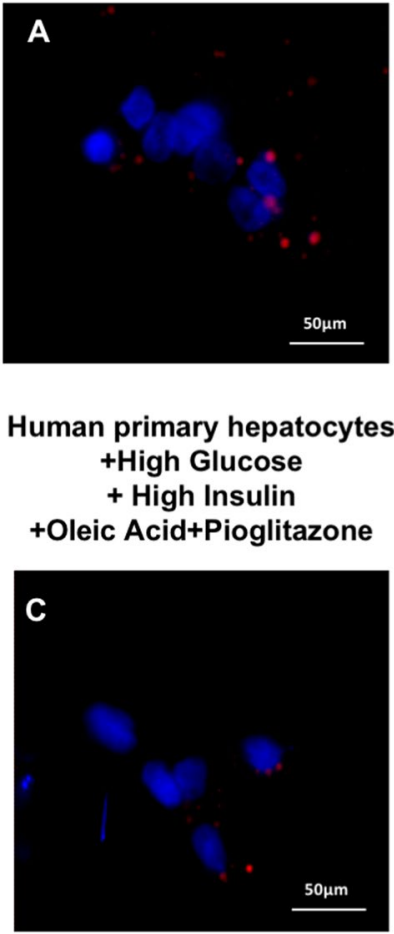

\section{Human primary hepatocytes \\ + High Glucose \\ + High Insulin \\ + Oleic Acid}

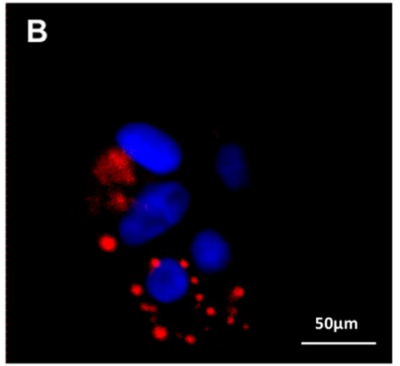

\section{Human Primary hepatocytes \\ +High Glucose \\ + High Insulin \\ +Oleic Acid+Metformin}

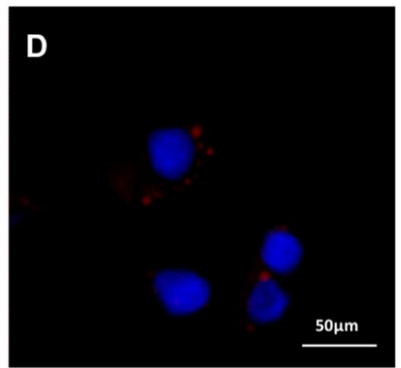

Figure 7. Pioglitazone or metformin decreased lipid droplet accumulation in human primary hepatocyte cultures. Panel (A): Nile Red staining of untreated hepatocyte primary culture. Panel (B): Nile Red staining of primary hepatocytes cultured for $24 \mathrm{~h}$ in the presence of high levels of oleic acid, glucose and insulin. Panel (C): Nile Red staining of primary hepatocytes cultured for $24 \mathrm{~h}$ in the presence of high levels of oleic acid, glucose, insulin and pioglitazone. Panel (D): Nile Red staining of primary hepatocytes cultured for $24 \mathrm{~h}$ in the presence of high levels of oleic acid, glucose, insulin and metformin.

hepatitis ${ }^{8}$, cholestatic liver disease ${ }^{9}$, presence of major cardiovascular, gastrointestinal, or respiratory disease, or any hormonal disorders ${ }^{10}$, clinically evidence of decompensated liver disease (Child-Pugh score $>7$ points) ${ }^{11}$, active substance abuse ${ }^{12}$, significant systemic illnesses ${ }^{13}$, pregnant status $^{14}$, type 2 diabetes.

The study was approved by the Ethical Committee of the University of Rome "La Sapienza" and the patients signed an informed consent prior the study enrolment; in addition, they signed a specific informed consent for the surgical operations and all methods were performed in accordance with the relevant guidelines and regulations

Human sleeve gastrectomy. Sleeve Gastrectomy (SG) consists in a vertical resection of the stomach in order to obtain a residual gastric capacity ranged from 60 to $80 \mathrm{ml}$. The procedures were carried out by laparoscopy using 5 trocars. The resection was performed using linear stapler with sequential cartridges alongside an oro-gastric calibration tube placed against the lesser curvature. Resection was begun 4 to $6 \mathrm{~cm}$ proximal to the pylorus and was continued up-ward to the angle of His with complete excision of the fundus, part of the body and antrum.

During the first month after surgery patients had a semiliquid diet with about $850 \mathrm{kcal}$ per day for the first month and thereafter they gradually introduced solid foodstuffs having a free diet.

Liver histology. Human liver biopsy material, obtained during the operation, was mounted on archival slides originally prepared from $4 \%$ formaldehyde-fixed paraffin-embedded tissue and stained with hematoxylin-eosin to evaluate the percentage of steatosis. Blind analysis of biopsy specimens was performed by a liver pathologist (CG).

We used the Brunt classification ${ }^{36}$ for the histological grading and staging of NAFLD/NASH. In particular, steatosis was graded as 0 , none $(<1 \%) ; 1,1-25 \% ; 2,26-50 \% ; 3,51-75 \%$; and $4,>75 \%$ amount of fat in the lobules. Inflammation was graded as 1 , mild (scattered lymphocytes or small clusters within portal tracts and lobules); 2, moderate (same as grade 1 but with a larger portal and lobular infiltration); and 3, severe (the same as grade 2 but with more intense inflammation). Fibrosis was staged as 0 if absent, 1 if centrilobular pericellular; 2 , if periportal and pericellular; 3 , bridging fibrosis; and 4, cirrhosis. 


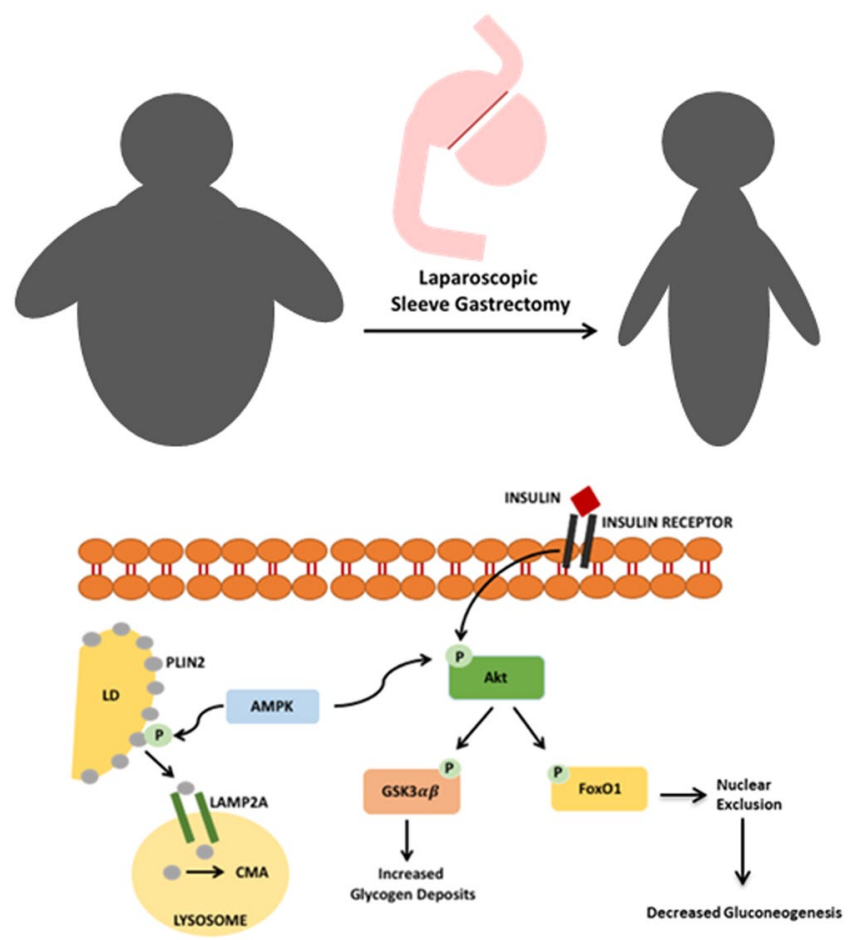

Figure 8. Proposed mechanism of NAFLD and insulin resistance reversal after Sleeve Gastrectomy. Excessive caloric intake, associated with NAFLD and obesity, reduces AMPK activation, causing a decrease of Plin2 catabolism mediated by chaperone-mediated autophagy leading to ectopic fat deposition increase and simultaneously to hepatic insulin resistance. After SG, the energy imbalance due to reduced caloric intake causes an increase in AMPK phosphorylation, a crucial step in Plin2-LAMP2A binding, leading to an enhanced Plin2 autophagy that exposes LD triglycerides to intracellular lipases. In addition, AMPK increased phosphorylation improves insulin signaling and promotes glycogen synthesis.

Insulin sensitivity. Hepatic insulin sensitivity was measured by the homeostasis model assessment (HOMA-IR) as fasting insulin $(\mu \mathrm{U} / \mathrm{ml}) \mathrm{x}$ fasting glucose $(\mathrm{mmol} / \mathrm{ml}) / 22.5^{37}$.

Lipid staining and glycogen storage. Periodic acid Schiff staining was used to evaluate glycogen storage. Slides were fixed 20 minutes with $4 \%$ formaldehyde, stained in Periodic Acid Solution for 5 minutes and in Schiff's Reagent for 15 minutes. Counterstain was performed with Hematoxylin solution.

Oil Red O was performed to assess intracellular lipid accumulation. Slides were fixed overnight with $4 \%$ formaldehyde, stained with Oil Red O solution for 1 hour. Counterstain was performed with Hematoxylin solution. Photographs of stained sections were taken with an optical microscope (ZEISS Primo Star HAL/LED).

Monocytes isolation. PBMCs were obtained from whole blood samples by standard gradient centrifugation over Ficoll-Hypaque. PBMCs were washed and monocytes were isolated following Pan Monocyte Isolation Kit instruction.

Human primary hepatocytes isolation. Hepatocytes from obese, NAFLD subjects and healthy subjects were isolated as described elsewhere ${ }^{38}$. Briefly, tissue was diced and washed in HBSS to remove excess blood. Tissue was transferred to a $50 \mathrm{ml}$ tube containing EGTA buffer (HBSS, $0.5 \mathrm{micromol} / \mathrm{L}$ EGTA, $0.5 \%$ BSA) and agitated at $100 \mathrm{rpm}$ in a water bath with shaking bed for $10 \mathrm{~min}$ at $37^{\circ} \mathrm{C}$. Tissue was then placed in digestion buffer (HBSS, $0.05 \%$ collagenase IV, $0.5 \%$ fatty acid free BSA, $10 \mathrm{micromol} / \mathrm{L} \mathrm{CaCl} 2$ ) and agitated in a water bath with shaking bed for $30 \mathrm{~min}$ at $37^{\circ} \mathrm{C}$. Supernatant was collected and filtered through $100 \mu \mathrm{m}$ cell strainer and the cell suspension centrifuged at $80 \mathrm{~g}$ for $5 \mathrm{~min}$ at $4^{\circ} \mathrm{C}$ and the supernatant discarded. Cells were cultured in DMEM in collagen coated dish at $37^{\circ} \mathrm{C}$ and $5 \% \mathrm{CO}_{2}$.

Antibodies and reagents. Antibodies against phospho-Akt (Ser473), phospho-GSK3 $\alpha \beta$ (Ser21/9), pospho-AMPK(Thr172) and phospho-FoxO1 (Thr24) were obtained from Cell Signaling Technology (Danvers, MA). Plin2 antibody was obtained from LS-BIO (Seattle, WA). LAMP2A was obtained from Thermo Fisher scientific (Waltham, MA). $\beta$-actin antibody was obtained from Santa Cruz Technology (Santa Cruz, CA). Period Acid, Schiff's reagent, Oil red O, Nile red, DAPI and Hematoxylin were obtained from Merck (Darmstadt, DE). 
Western blot analysis. Monocytes and liver biopsies were homogenized in RIPA buffer containing a cocktail of protease inhibitors. Homogenates were cleared by centrifugation $\left(19.000 \mathrm{~g} ; 30 \mathrm{~min}, 4^{\circ} \mathrm{C}\right)$. Protein content was determined using Bradford Protein Assay. Protein lysates $(30 \mu \mathrm{g})$ were separated on $10 \%$ SDS-PAGE and transferred on PVDF membrane ${ }^{33,39}$. Membranes were probed overnight with Plin2, phospho-AktSer473, GSK3 $\alpha \beta$ Ser21/9, FoxO1 Thr24, AMPK Thr172, LAMP2A, and $\beta$ Actin. Membranes with phospho-antibodies were stripped for $30 \mathrm{~min}$ at $56^{\circ} \mathrm{C}$ and reprobed overnight with total Akt, GSK3 $\alpha-\beta$, AMPK and FoxO1respectively. Detection and analysis were performed respectively with Chemidoc XRS Image system and Image Lab 5.0 software (Bio-Rad Laboratories, Hercules, CA) ${ }^{33,39}$. Plin2 and LAMP2A were normalized with $\beta$ Actin while phospho-AktSer473, GSK3 $\alpha \beta$ Ser21/9, FoxO1 Thr24 and AMPK Thr172 were normalized with total Akt, GSK $3 \alpha-\beta$, FoxO1, AMPK respectively and expressed as a phospho-protein/total protein ratio.

\section{In vitro Study}

Lipid droplet accumulation. Primary hepatocytes and monocytes were stimulated with Oleic Acid (400 $\mu \mathrm{mol} / \mathrm{L})$ for 24 hours and stained with Nile Red $(100 \mathrm{ng} / \mathrm{mL})$. Flow cytometric analysis and confocal imaging were conducted to quantify lipid droplets. Flow cytometric analysis was conducted with FC 500 (Beckman Coulter, Brea, CA) and data were analyzed with Kaluza software (Beckman Coulter, Brea, CA). Photographs were taken using confocal microscope Spinning Disk; Crest X-Light Confocal Imager (Germany) and MetaMorph Microscopy Automation \& Image Analysis Software (Molecular Devices) was used to analyze images.

Quantitative data from flow cytometry analysis were used to assess the cellular content of LDs stained with Nile Red and used for the Bland Altman plot.

Flow cytometry. Monocytes and primary hepatocytes were washed and resuspended in PBS containing $5 \%$ FBS. Cells were fixed, permeabilized and stained for Plin2 using AlexaFluor 488 as secondary antibody, with CD14 to identify monocytes population and GLUT2 to identify hepatocytes. Flow cytometric analysis was conducted with FC 500 (Beckman Coulter, Brea, CA) and data were analyzed with Kaluza software (Beckman Coulter, Brea, CA).

Plin2 transfection. Primary hepatocytes and monocytes were transfected with human PLIN2 plasmid using Nucleofector ${ }^{\mathrm{TM}}$ Kits and following manufactures instruction. As a positive control for transfection we used $2 \mu \mathrm{g}$ pmaxGFP ${ }^{\circledR}$ Vector. Untrasfected cells and cells transfected with human Plin2 plasmid were culture for 6 hours. To evaluate the efficiency of transfection and Plin2 overexpression cells were analyzed with flow cytometry. To asses Plin2 overexpression effect on AktSer473, GSK3 $\alpha \beta$ Ser21/9 and FoxO1 Thr24 cells were analyzed by western blot.

Pioglitazone and metformin exposure. Primary hepatocytes were incubated for 24 hrs with insulin $(100 \mathrm{nmol} / \mathrm{L})$, Glucose $(25 \mathrm{mmol} / \mathrm{L})$, Oleic Acid $(400 \mu \mathrm{mol} / \mathrm{L})$ and with or without pioglitazione $(25 \mu \mathrm{mol} / \mathrm{L})$ or metformin $(2 \mathrm{mmol} / \mathrm{L})$. Western blot analysis were performed to assess AMPK Thr172, LAMP2A and Plin2 expression.

Lipid staining. Primary hepatocytes were incubated for 45 minutes at $37^{\circ} \mathrm{C}$ and $5 \% \mathrm{CO}_{2}$ with Nile Red (100 ng/mL). Nuclear staining was performed with DAPI.

Photographs were taken using confocal microscope Spinning Disk; Crest X-Light Confocal Imager (Germany) and MetaMorph Microscopy Automation \& Image Analysis Software (Molecular Devices) was used to analyze images.

Statistical analysis. Data were expressed as means \pm s.e.m. unless specified otherwise. Statistical analyses (SPSS version 13) were performed using Mann-Whitney or Wilcoxon tests and repeated measures test with Bonferroni's correction or Dunn's Multiple Comparison test where appropriate. Differences were considered statistically significant at $\mathrm{P} \leq 0.05$.

\section{Data availability}

The authors declare that all the data supporting the findings of this study are available in the manuscript, figures and supplementary information files.

Received: 24 May 2019; Accepted: 18 September 2019;

Published online: 21 November 2019

\section{References}

1. Younossi, Z. M. et al. Global epidemiology of nonalcoholic fatty liver disease-Meta-analytic assessment of prevalence, incidence, and outcomes. Hepatology. 64, 73-84 (2016).

2. Loomis, A. K. et al. Body Mass Index and Risk of Nonalcoholic Fatty Liver Disease: Two Electronic Health Record Prospective Studies. J. Clin. Endocrinol. Metab. 101, 945-952 (2016).

3. Birkenfeld, A. L. \& Shulman, G. I. Nonalcoholic fatty liver disease, hepatic insulin resistance, and type 2 diabetes. Hepatology. 59, 713-723 (2014).

4. Mingrone, G. et al. Bariatric surgery versus conventional medical therapy for type 2 diabetes. N. Engl. J. Med. 366, 1577-1585 (2012).

5. Schauer, P. R. et al. Bariatric surgery versus intensive medical therapy in obese patients with diabetes. N. Engl. J. Med. 366, 1567-1576 (2012).

6. Leonetti, F. et al. Obesity, type 2 diabetes mellitus, and other comorbidities: a prospective cohort study of laparoscopic sleeve gastrectomy vs medical treatment. Arch. Surg. 147, 694-700 (2012).

7. Schauer, P. R. et al. STAMPEDE Investigators. Bariatric Surgery versus Intensive Medical Therapy for Diabetes - 5-Year Outcomes. N. Engl. J. Med. 376, 641-651 (2017). 
8. Ikramuddin, S. et al. Lifestyle Intervention and Medical Management With vs Without Roux-en-Y Gastric Bypass and Control of Hemoglobin A1c, LDL Cholesterol, and Systolic Blood Pressure at 5 Years in the Diabetes Surgery Study. JAMA. 319, 266-278 (2018).

9. Mingrone, G. et al. Bariatric-metabolic surgery versus conventional medical treatment in obese patients with type 2 diabetes: 5 year follow-up of an open-label, single-centre, randomised controlled trial. Lancet. 386, 964-973 (2015).

10. Castagneto Gissey, L. et al. 10-year follow-up after laparoscopic sleeve gastrectomy: Outcomes in a monocentric series. Surg. Obes. Relat. Dis. 14, 1480-1487 (2018).

11. Lassailly, G. et al. Bariatric Surgery Reduces Features of Nonalcoholic Steatohepatitis in Morbidly Obese Patients. Gastroenterology. 149, 379-388 (2015).

12. Garcia, D. \& Shaw, R. J. AMPK: Mechanisms of Cellular Energy Sensing and Restoration of Metabolic Balance. Mol. Cell. 66, 789-800 (2017).

13. Hardie, D. G., Ross, F. A. \& Hawley, S. A. AMPK: a nutrient and energy sensor that maintains energy homeostasis. Nat. Rev. Mol. Cell. Biol. 13, 251-262 (2012).

14. Ezquerro, S. et al. Acylated and desacyl ghrelin are associated with hepatic lipogenesis, $\beta$-oxidation and autophagy: role in NAFLD amelioration after sleeve gastrectomy in obese rats. Sci Rep. 6, 39942 (2016).

15. McIntosh, A. L. et al. Direct interaction of Plin2 with lipids on the surface of lipid droplets: a live cell FRET analysis. Am. J. Physiol. Cell. Physiol. 303, 728-742 (2012).

16. Libby, A. E., Bales, E., Orlicky, D. J. \& McManaman, J. L. Perilipin-2 Deletion Impairs Hepatic Lipid Accumulation by Interfering with Sterol Regulatory Element-binding Protein (SREBP) Activation and Altering the Hepatic Lipidome. J. Biol. Chem. 291, 24231-24246 (2016).

17. Chang, B. H. et al. Protection against fatty liver but normal adipogenesis in mice lacking adipose differentiation-related protein. Mol. Cell. Biol. 26, 1063-1076 (2016).

18. Kaushik, S. \& Cuervo, A. M. Degradation of lipid droplet-associated proteins by chaperone-mediated autophagy facilitates lipolysis. Nat. Cell. Biol. 17, 759-770 (2015).

19. Kaushik, S. \& Cuervo, A. M. AMPK-dependent phosphorylation of lipid droplet protein PLIN2 triggers its degradation by CMA. Autophagy. 12, 432-438 (2016).

20. Carpentier, J. L., Gorden, P., Freychet, P., Le Cam, A. \& Orci, L. Lysosomal association of internalized 125I-insulin in isolated rat hepatocytes. Direct demonstration by quantitative electron microscopic autoradiography. J Clin Invest. 6, 1249-61 (1979).

21. Frühbeck, G. Bariatric and metabolic surgery: a shift in eligibility and success criteria. Nat Rev Endocrinol. 8, 465-77 (2015).

22. Cokorinos, E. C. et al. Activation of Skeletal Muscle AMPK Promotes Glucose Disposal and Glucose Lowering in Non-human Primates and Mice. Cell Metab. 25, 1147-1159 (2017).

23. Fullerton, M. D. et al. Single phosphorylation sites in Acc1 and Acc2 regulate lipid homeostasis and the insulin-sensitizing effects of metformin. Nat Med. 19, 1649-1654 (2013).

24. Myers, R. W. et al. Systemic pan-AMPK activator MK-8722 improves glucose homeostasis but induces cardiac hypertrophy. Science. 357, 507-511 (2017).

25. Steneberg, P. et al. PAN-AMPK activator O304 improves glucose homeostasis and microvascular perfusion in mice and type 2 diabetes patients. JCI Insight. 3, e99114 (2018).

26. Boudaba, N. et al. AMPK Re-Activation Suppresses Hepatic Steatosis but its Downregulation Does Not Promote Fatty Liver Development. EBioMedicine. 28, 194-209 (2018).

27. Cool, B. et al. Identification and characterization of a small molecule AMPK activator that treats key components of type 2 diabetes and the metabolic syndrome. Cell Metab. 3, 403-416 (2006).

28. Garcia, D. et al. Genetic Liver-Specific AMPK Activation Protects against Diet-Induced Obesity and NAFLD. Cell Rep. 26, 192-208 (2019).

29. Perry, R. J., Samuel, V. T., Petersen, K. F. \& Shulman, G. I. The role of hepatic lipids in hepatic insulin resistance and type 2 diabetes. Nature. 510, 84-91 (2014)

30. Lamontagne, J. et al. Pioglitazone acutely reduces energy metabolism and insulin secretion in rats. Diabetes 62, 2122-2129 (2013).

31. Ezquerro, S. et al. Ghrelin Reduces TNF- $\alpha$-Induced Human Hepatocyte Apoptosis, Autophagy, and Pyroptosis: Role in ObesityAssociated NAFLD. J Clin Endocrinol Metab. 104(1), 21-37 (2019).

32. Radziuk, J. Insulin sensitivity and its measurement: structural commonalities among the methods. J. Clin. Endocrinol. Metab. 85, 4426-4433 (2000).

33. Casella-Mariolo, J. et al. Simulation of gastric bypass effects on glucose metabolism and non-alcoholic fatty liver disease with the Sleeveballoon device. EBioMedicine. 46, 452-462 (2019).

34. Mohamad, Y. R. \& Hany, A. E. Kisspeptin and Vaspin: Indicators of Insulin Sensitivity Improvement before Weight Loss Following Sleeve Gastrectomy in Experimental Type 2 Diabetes Mellitus. Int J Diabetes Res. 6(4), 91-98 (2017).

35. Papeleu, P. et al. Isolation of rat hepatocytes. Methods. Mol. Biol. 320, 229-237 (2006).

36. Kleiner, D. et al. Design and validation of a histological scoring system for nonalcoholic fatty liver disease. Hepatology. 41, 1313-1321 (2005).

37. Matthews, D. R. et al. Homeostasis model assessment: insulin resistance and beta-cell function from fasting plasma glucose and insulin concentrations in man. Diabetologia. 28, 412-419 (1985).

38. Green, C. J. et al. The isolation of primary hepatocytes from human tissue: optimising the use of small non-encapsulated liver resection surplus. Cell Tissue Bank. 18, 597-604 (2017).

39. Angelini, G., Salinari, S., Bertuzzi, A., Iaconelli, A. \& Mingrone, G. Metabolic surgery improves insulin resistance through the reduction of gut-secreted heat shock proteins. Commun Biol. 13(1), 69 (2018).

\section{Acknowledgements}

We thank Mrs Anna Caprodossi and Dr. Alessandra Ciucci for their technical assistance. Professor G. Casella: Linea di Ricerca Università La Sapienza 2016. Professor G. Mingrone: Linea di Ricerca D1 UCSC 2017. Professor G. Mingrone, Professor S. Bornstein, Professor A. Birkenfeld: DFG - IRTG 2251.

\section{Author contributions}

Design of the work: G.A. and G.C.; acquisition of the data: L.C.G., G.DC., C.G. and B.C.; analytical methods: G.A. and A.S.; analysis of data: G.A., G.C. and L.C.G.; interpretation of data and manuscript writing: G.A., G.C., G.M., A.L.B., S.R.B., A.G., M.M. and N.B.

\section{Competing interests}

The authors declare no competing interests. 


\section{Additional information}

Supplementary information is available for this paper at https://doi.org/10.1038/s41598-019-53702-4.

Correspondence and requests for materials should be addressed to G.C.

Reprints and permissions information is available at www.nature.com/reprints.

Publisher's note Springer Nature remains neutral with regard to jurisdictional claims in published maps and institutional affiliations.

(c) (i) Open Access This article is licensed under a Creative Commons Attribution 4.0 International License, which permits use, sharing, adaptation, distribution and reproduction in any medium or format, as long as you give appropriate credit to the original author(s) and the source, provide a link to the Creative Commons license, and indicate if changes were made. The images or other third party material in this article are included in the article's Creative Commons license, unless indicated otherwise in a credit line to the material. If material is not included in the article's Creative Commons license and your intended use is not permitted by statutory regulation or exceeds the permitted use, you will need to obtain permission directly from the copyright holder. To view a copy of this license, visit http://creativecommons.org/licenses/by/4.0/.

(c) The Author(s) 2019 